\title{
Pharmacotherapy in the management of early Parkinson's disease: cost-effectiveness and patient acceptability
}

This article was published in the following Dove Press journal:

ClinicoEconomics and Outcomes Research

4 September 2010

Number of times this article has been viewed

\author{
Esther Cubo \\ Neurology Department, Hospital \\ General Yagüe, Burgos, Spain
}

Correspondence: Esther Cubo Neurology Department, Hospital General Yagüe, Avda Cid 96, 09005 , Burgos, Spain

Tel +34948281922

$\mathrm{Fax}+34$ 947281965

Email esthercubo@gmail.com

\begin{abstract}
In the absence of a cure, the primary goals in managing Parkinson's disease (PD) are to preserve functionality and health-related quality of life (HRQoL). Current therapeutic strategies for PD include symptomatic treatment and are primarily focused on replacing dopamine in the brain. Dopamine agonists can be used as an alternative initial levodopa therapy, to delay the onset of motor complications, but at the expense of more dopaminergic adverse effects; poorer control of motor symptoms; and increased cost. In PD, treatment effects and costs accumulate over time; hence the choice of time horizon in cost-effectiveness analysis can be particularly important. Pharmaceutical expenditures have grown rapidly in recent decades and now total nearly $10 \%$ of all health care costs. The main approach to treat PD at the present time is to advance knowledge of the efficacy, to reduce long-term complications associated with treatment, and to improve patient HRQoL and society burden. The implementation of cost-effectiveness studies, including the societal perspective, should be considered as an outcome of new therapy strategies, which would be helpful to health care decision makers.
\end{abstract}

Keywords: Parkinson's disease, costs, health-related quality of life

\section{Introduction}

Parkinson's disease (PD) is an age-related illness, primarily affecting the elderly population and often resulting in a marked decline in the health-related quality of life (HRQoL) of both patients and caregivers. ${ }^{1,2}$ Data from several studies show that PD represents a serious health problem, resulting in increased health expenditures and loss of productivity. ${ }^{3}$ In view of current demographic trends; the progressive and long-term nature of PD; and the proportion of people with the disease, the ensuing use of health care resources will increase. ${ }^{4-8}$ Current therapeutic strategies for PD include symptomatic treatment which is primarily focused on replacing dopamine in the brain. As levodopa has provided the greatest symptomatic benefit with the fewest short-term adverse effects, it is still considered the gold standard of symptomatic treatment. However, long-term levodopa is associated with fluctuations in motor performance, dyskinesias, and neuropsychiatric complications..$^{910}$ Dopamine agonists can be used as an alternative initial therapy, to delay the onset of motor complications, but at the expense of more dopaminergic adverse effects; poorer control of motor symptoms; and increased cost. ${ }^{11}$ Thus, the main approach to treat PD at the present time is to advance knowledge of the efficacy, to reduce long-term complications associated with treatment and to improve patient HRQoL and society burden.

Cost effectiveness studies of PD therapeutic interventions are therefore essential to help the decision making of care providers, affected individuals and their families, 
and policy makers, and also for predicting alternative interventions. Therefore, the main purpose of this review was to evaluate the current knowledge of cost-effectiveness and patient acceptability of pharmacotherapy and other interventions in early PD.

\section{Effectiveness outcomes Clinical effectiveness outcomes}

In order to evaluate the clinical effectiveness of PD interventions, most clinical trials use surrogate endpoints or focus on PD symptoms severity, complications, or impact on patient HRQoL. However, assessing clinical effectiveness outcomes is highly complex due to the wide spectrum of PD symptoms, complications, and lifelong progression of the disease. In this regard, several different rating scales have been developed to assess various effectiveness outcomes in PD patients. The first simple descriptive staging scale for PD was that of Hoehn and Yahr (HY), ${ }^{12}$ designed to provide a general estimate of clinical function in $\mathrm{PD}$, based on the concept that the severity of PD was related to bilateral motor dysfunction and impairment of gait and balance (ranging from Stage I, with unilateral presence of motor symptoms; to Stage V, being wheelchair- or bed-bound). However, because the broad categories of the HY scale do not allow consistent identification of effective interventions, ${ }^{13}$ the scale that has been largely used in clinical trials is the Unified Parkinson's Disease Rating Scale (UPDRS),${ }^{14}$ and its new version, the Movement Disorder Society-sponsored revision of the Unified Parkinson's Disease Rating Scale (MDS-UPDRS), ${ }^{15}$ designed to accommodate new advances and to resolve problematic areas. The MDS-UPDRS has four parts: I - Non-motor Experiences of Daily Living; II - Motor Experiences of Daily Living; III - Motor Examination; IV - Motor Complications. Twenty questions are completed by the patient/caregiver. The published data supports the validity of this scale for rating PD.

As important as economic costs are to any discussion of PD-related resource utilization, it is also critical that payers and providers consider the significant impact the disease has on HRQoL, which assesses an individual's perceived effect of the illness on their physical, psychological, and social daily lives. It is important for determining the effectiveness of therapies for PD at both individual and population levels. For care provider managers, it presents an important parameter to measure the effectiveness of management strategies and quality of care. HRQoL measures are also important in assessing the value of drug therapy, particularly for chronic conditions such as $\mathrm{PD}$, and in determining the appropriate placement of medications on plan formularies. As would be expected for any chronic and progressively worsening disorder, PD has a significant impact on the HRQoL of both patients and their caregivers. Of note, non-motor disability (particularly depression, insomnia, and other mental health factors) appears to have a greater negative effect on HRQoL than motor deficits. ${ }^{16,17}$ The most common generic health profile measures to evaluate PD are the Nottingham Health Profile (NPH), ${ }^{18,19}$ the 36-Item Short Form General Health Survey (SF36), ${ }^{20}$ and the Sickness Impact Profile (SIP). ${ }^{21}$ Utility measures used include the EQ-5D ${ }^{\mathrm{TM}}$ and the Health Utility Index..$^{22,23}$ However, most of the cost-effectiveness analyses have used the EQ-5D ${ }^{22}$ as a measurement of HRQoL, or quality-adjusted life years (QALYs). The EQ-5D consists of five questions (mobility, self care, usual activities, pain, and anxiety/depression) and an additional visual analog scale on which patients rate their current health status. QALYs, or years of healthy life, are used instead in order to quantify time and health. The QALY concept comprises two qualities: the first, the time component that considers the gain or loss of life time due to the choice of a certain treatment strategy; and the second, the HRQoL is measured by its value on a scale from 0 (death) to 1 (perfect health). ${ }^{13}$ Thus, one QALY means a period of 1 year under the condition of perfect health. On the other hand, disease-specific instruments are usually more responsive to a change in health status of a particular patient group compared with generic instruments. ${ }^{24} \mathrm{HRQoL}-$ specific measures of PD include the PDQ-39, ${ }^{25}$ the Parkinson's disease quality of life questionnaire (PDQL), ${ }^{26,27}$ and the Parkinson's Impact Scale (PIMS). ${ }^{28}$

\section{Cost-effectiveness of treatment options}

In cost-of-illness studies, three types of costs are evaluated, including direct; indirect; and intangible costs. Direct costs arise directly from the treatment of the disease, while indirect costs refer to indirect consequences of the disease, such as loss of work and early retirement. Intangible costs are costs that cannot be directly expressed in monetary values, such as happiness or anxiety due to a disease. Another controversially discussed cost component to consider in cost analysis studies is informal care (care by volunteers such as family members or friends), because from the perspective of a third-party payer, the costs of informal care are irrelevant (as are indirect costs). They cannot be reimbursed, but considered from a societal perspective, they should be legitimately included. ${ }^{13}$ McCrone et al, using a regression analysis, identified significant baseline predictors 
of follow-up costs in PD. ${ }^{29}$ According to these authors, formal service costs accounted for $20 \%$ of this figure, with informal care from families/friends accounting for $80 \%$. The regression model explained $42 \%$ of total follow-up costs, and significant predictors included gender (with men having higher costs), disability, and depression. However, the majority of authors conclude that in $\mathrm{PD}$, along with costs for hospitalization, drug costs are consistently the main cost driver of direct costs. ${ }^{13,30,31}$ Direct and indirect costs related to PD increase with the progression of the disease. Over time, the direct costs of patients with predominant akineticrigid $\mathrm{PD}$ phenotype are higher than those with predominant tremor, and unaffected peers. ${ }^{32}$

There are different types of economic evaluations, but cost-effectiveness analysis and cost-utility analysis are usually the preferred analyses that help decision makers in the allocation of restricted health care resources. ${ }^{13}$ Cost-effectiveness analysis measures the benefit of a health intervention in relation to costs, and represents the cost of the treatment option in monetary units by the expected effectiveness measures in natural units, such as, for example, UPDRS score decrease. ${ }^{33,34}$ However the main critique of this type of evaluation is the difficulty of comparing between different patient populations with different diseases. ${ }^{13}$ Instead, to compare interventions for different diseases, one has to use a generic denominator such a QALYs, which can be calculated on the basis of the patient's subjectively assessed HRQoL, and are based on patient preferences. This is called cost-utility analysis. The latter gives us the additional amount of money one has to pay for therapeutic interventions to gain one more QALY. ${ }^{13}$

For health maker decisions, two approaches have frequently been used: decision trees and Markov models. In decision trees, one decision node and its roots and all branches of the initial decision node are represented as treatment options that are to be compared. This type of approach is useful if the outcomes to analyze are short-term, the duration of the disease is short, and if events within the course of a disease do not occur repeatedly. ${ }^{13}$ Markov models, on the other hand, allow for the synthesis of data on cost, effects, and HRQoL of alternative clinical strategies, by assigning values to a series of health states over time.

\section{Cost-effectiveness in early PD}

Over the last years, a growing number of randomized controlled trials of medical therapies have included economic evaluations and HRQoL as secondary outcomes. These kinds of evaluations can provide data about the value of a drug or technique when it is introduced into the market. A search of the published literature from January 1st 1990 to February 15th 2010 was undertaken using the keywords: "Parkinson"; "Parkinson's disease"; "cost"; "cost effectiveness"; and "quality of life", in combination. Databases searched were PubMed and the Cochrane Library. Only English language publications which included patients with early $\mathrm{PD}$, defined as having disease duration less than two years, were considered.

\section{Cost-effectiveness of new techniques to diagnose PD}

Diagnostic errors occur in $10 \%-25 \%$ of $\mathrm{PD}$ patients managed by general neurologists, leading to delayed or inappropriate therapy initiation. ${ }^{35,36}$ New imaging techniques use radioactive tracers, such as 123I-ioflupane (123I-FP-CIT), to provide earlier and more accurate diagnosis than that of the standard clinical assessment. ${ }^{37}$ The 123I-FP-CIT tracer binds to the striatal dopamine transporter (DAT) and is used in combination with single photon emission computed tomography (SPECT) to identify dopaminergic deficits. ${ }^{36}$ The economic advantages associated with the use of 123I-FP-CIT SPECT (DaTSCAN) may vary depending on the prevalence of PD in the group of patients with uncertain diagnosis; the cost of routine clinical evaluation including other diagnostic tests; and the consequences of either delaying or inappropriately instituting dopaminergic therapy. ${ }^{38}$ In one recent study by Antonini et al, conducted from an Italian National Health System perspective, DaTSCAN was compared to clinical judgment alone for differentiating essential tremor from $\mathrm{PD} .^{37}$ Based on the Markev models, over 5 years, the estimated cost-effectiveness of DaTSCAN was under 1000 Euros (€) per potentially beneficial therapy (2005 values) gained when the underlying disease prevalence is high (55\%-70\%), and cost-saving at a prevalence under $55 \%$. These authors concluded that DaTSCAN is likely to be economically advantageous to differentiate essential tremor from $\mathrm{PD}$, increasing time on potentially beneficial therapy, at lower overall cost to the health care system. ${ }^{37}$ However further studies are required to validate these results in different national health care systems.

\section{Cost-effectiveness of dopaminergic drugs}

Pharmacological treatments have been directed primarily at dopamine replacement with levodopa and agents to improve its bioavailability. These include DOPA decarboxylase inhibitors; catechol-O-methyltransferase (COMT) inhibitors; and monoamine oxidase $\mathrm{B}$ (MAO-B) inhibitors, as well as 
synthetic dopamine agonists. There is still uncertainty as to when to start medical treatment in PD. The issue of whether to adopt a "wait and watch" strategy or to initiate drug therapy soon after diagnosis in PD has been the subject of some debate. A recent observational study supported early treatment by demonstrating deterioration in self-reported health status in those left untreated, but not those who received therapy. ${ }^{39}$ There is therefore increasing evidence of a negative influence on disease progression by delayed onset of medical drug treatment in PD. However, on the other hand, it seems that drug treatment in early PD patients increases health values, but the incremental cost-effectiveness ratio is high. ${ }^{40}$ Greater awareness of a prodromal/pre-motor stage of the disease; efforts toward early and accurate diagnosis; and the continuous refinement of treatment paradigms, provide an opportunity for discussion on the use of potential disease-modifying agents to slow or halt the progression of motor and non-motor disability. Such compounds could not only significantly improve patient and caregiver quality of life, but substantially reduce direct and indirect costs.

To date, numerous compounds have been evaluated in clinical trials, including coenzyme Q10; creatine; levodopa; pramipexole; rasagiline; ropinirole; and selegiline. None have demonstrated irrefutable and enduring disease-modifying qualities, although the best available clinical evidence appears most promising for rasagiline. Another important issue to be considered is that, although effective treatment of motor symptoms of PD is a central consideration to facilitate improved outcomes, management of non-motor symptoms is an equally, still to be improved, important target of intervention, since these symptoms can contribute greatly to disability.

We studied the impact of motor and non-motor symptoms on PD direct costs based on the Spanish National Health System (2004€ values). ${ }^{31}$ Average three month total direct cost of PD was 2,631 $\pm 4,507 €$ per person (range: 71,5-29,159) and medical treatment was the main cost driver, accounting for $34 \%$ of the cost (mean drug cost per person: $669 \pm 406 €$ ). PD direct costs were significantly higher among younger patients, with higher disease severity, motor impairment, complications and had a higher impact on the direct costs of PD than non-motor symptoms. This finding can be at least partially explained due to the lack of effective treatment for the majority of non-motor symptoms.

As dopaminergic drugs can also be used for PD and restless legs syndrome, the review of cost-analysis of dopaminergic drugs included in this article applied to PD only. In this review, because ergot agonists are no longer in use, and in agreement with the published literature, only first-line treatment for early PD was included (selegiline, rasagiline, pramipexole, and ropinirole). No cost-effectiveness studies for rotigotine were identified at the present time.

\section{Selegiline}

Selegiline, the first selective inhibitor of monoamine oxidase type $\mathrm{B}$ inhibitor (MAO-B), described in the literature, is a selective, irreversible cerebral that is used in the treatment of PD. It has a relatively mild adverse effect profile, with no risk of the tyramine ('cheese') reaction at normal therapeutic doses. Selective MAO-B inhibitors, used as monotherapy, delay the need for the introduction of levodopa by about nine months, although the relative contribution of neuroprotective and symptomatic effects of selegiline in these patients has yet to be clarified. These agents appear to be less efficacious than dopamine agonists, but are better tolerated. Concern has been expressed about the potential of the MAO-B inhibitor selegiline to induce cardiovascular adverse effects (orthostatic hypotension), either directly or through its amphetamine catabolites. ${ }^{41}$ From a societal perspective, an old study concluded that the economic benefits of selegiline therapy are likely to be substantial. ${ }^{42}$ An agent which slowed progression of disability by around 10\% would realize savings, through reduction in both direct and indirect costs, in the order of 330 million US dollars (USD) per annum in the United States (1992 USD values). ${ }^{42}$ No other studies could be identified comparing selegiline with dopaminergic agonists, which are the preferred treatment option of patients with early PD without comorbidities, or with the other MAO-B inhibitor, rasagiline.

\section{Rasagiline}

Rasagiline is a second-generation MAO-B inhibitor that selectively and irreversibly inhibits brain MAO-B, specifically designed for the treatment of PD. In one recent study, the cost effectiveness, from a United Kingdom health care payer perspective, of two anti-Parkinsonian treatment strategies in early PD (first-line monotherapy with rasagiline; and the non-ergoline dopamine receptor agonist pramipexole) was analyzed over a 5-year period, using an economic Markov model. ${ }^{45}$ Utility and costs of these two strategies and effectiveness outcomes were: i) time to levodopa; and ii) time to levodopa-induced dyskinesia. These authors found that, compared with pramipexole, the use of the rasagiline strategy was estimated to reduce costs by $18 \%$ per patient over 5 years and was associated with an additional 10\% delay in dyskinesia onset (0.41 years; 95\% CI: $0.27-0.55)$. 
This strategy was also found to prolong the time to levodopa initiation by $25 \%$, through a gain of 0.83 levodopa-free years (95\% CI: 0.56-1.1). In addition, use of the rasagiline strategy was found to generate a 5\% gain in QALYs over 5 years, compared with the pramipexole strategy $(3.7 \pm 0.02$ vs $3.51 \pm 0.03) .{ }^{43}$ In addition, rasagiline seemed to improve HRQoL compared with placebo. ${ }^{44}$ This HRQoL improvement appears to be accounted for primarily by the symptomatic benefit of rasagiline. Interestingly, by using the PDQL questionnaire, most of this benefit was found in the self-image/ sexuality domain. However, further cost-effectiveness studies comparing rasagiline with other dopaminergic agonists are required.

\section{Pramipexole}

Evidence from pre-clinical studies and clinical trials has proven the effectiveness of pramipexole in ameliorating the symptoms of PD. There is also evidence in animal studies that this agent, a nonergoline dopaminergic agonist, may be neuroprotective and could therefore possibly slow disease progression; however, this has yet to be proven in humans. The use of pramipexole may be limited by its side effects profile compared to standard therapies and its relatively higher cost compared to levodopa. ${ }^{45}$ Different studies have been published looking at the cost-effectiveness of pramipexole with no levodopa therapy, compared to levodopa monotherapy and to rasagiline in early PD. ${ }^{43,46-49}$ Hoerger et $a l^{46}$ used mathematical equations to link UPDRS parts (II and III scores), to analyze costs and QALYs in a US setting. For patients with early PD, treatment with pramipexole had higher costs, but was more effective than baseline treatment. For patients with early onset of PD, the incremental total cost-effectiveness ratio for pramipexole was 8,837 US dollars (USD)/QALY and direct costs were USD 67,702 for pramipexole compared to USD 57,549 for the baseline treatment. On the other hand, costs of loss of productivity were USD 97,391 for pramipexole versus USD 104,937 for baseline treatment (1997 costings). The authors concluded that it indicated higher costs for patients with early PD in the US receiving pramipexole, than for those not taking the drug, but additional quality life-years were gained.

In one longitudinal study, the two-year incremental cost effectiveness of initial pramipexole treatment was compared with initial levodopa treatment in 301 subjects with early PD, randomized to either agent. ${ }^{47}$ Pramipexole strategy was an estimated USD 2,138 $\pm 1,182$ more expensive than levodopa strategy. The incremental cost-effectiveness of pramipexole compared with levodopa was USD 106,900/ QALY (EQ-5D), compared with pramipexole being dominated by levodopa using the EQVAS (2002 costings). After four years of follow-up, the cost-effectiveness of pramipexole compared with levodopa in the treatment of early PD increased as the time horizon of the clinical trial extended from two to four years. ${ }^{48}$ Using the costeffectiveness acceptability curves to estimate the probability that pramipexole was cost-effective, given different societal values of QALY, the probability that pramipexole was cost-effective relative to levodopa over the first four years was $0.57,0.77$ and 0.82 , when a QALY was valued at USD 50,000, USD 100,000, and USD 150,000 respectively (2002 USD costs). Over time, the incremental cost-effectiveness ratio for pramipexole improved and uncertainty around the incremental cost-effectiveness ratio decreased. Likewise, HRQoL improved in pramipexole subjects and declined in levodopa subjects, and the probability of pramipexole being cost-effective increased to $0.88,0.96$, and 0.98 respectively. The authors also found that pramipexole seemed to be more cost-effective for patients with depression and low baseline HRQoL. However, it seems that the methodology applied to evaluate cost-effectiveness had an impact on the result. One study by Noyes et $\mathrm{al}^{49}$ found that country-specific preference weights in clinical-economic trials might have important effects on estimates of incremental cost-effectiveness. Using US preference weights rather than UK preference weights reduced the probability that pramipexole was cost-effective compared to levodopa.

\section{Ropinirole}

Ropinirole is a non-ergoline dopamine agonist that binds to dopamine D2-receptors; the drug is indicated for use in the symptomatic treatment of early and late PD. In one costminimization study, conducted to examine the economic impact of reducing dyskinesias using ropinirole instead of levodopa plus benserazide in $\mathrm{PD},{ }^{50}$ the authors found, from the perspective of the Canadian Ministry of Health, that ropinirole was more expensive than levodopa. The analysis yielded an incremental expected daily cost/patient of 4.41 Canadian dollars (CAD) for substituting levodopa plus benserazide with ropinirole. On the other hand, from a societal perspective, in which loss of productivity was the major factor, ropinirole was cost-saving, after off-setting the drug costs. The added costs of ropinirole were counterbalanced by savings due to reduced dyskinesias and associated downstream costs were also examined (1999 CAD). The main criticism of this study was that important cost and 
resource-utilization data were estimated by experts, which can limit extrapolation. ${ }^{13}$

\section{Cost-effectiveness of nonpharmacological treatments}

Despite optimal medical management, most PD patients become progressively disabled. Allied health care usually provide complementary benefits to PD patients, even for symptoms that are resistant to pharmacotherapy or surgery. ${ }^{51}$ Clinical experience suggests that optimal management requires a multidisciplinary approach, with multifactorial health plans tailored to the needs of each individual patient. However, little is known about the cost-effectiveness of a multidisciplinary team approach. Since the first guideline on physical therapy published in $2004,{ }^{52}$ providing recommendations for evidence-based interventions, the quantity and quality of clinical trials evaluating the efficacy of physical therapy in PD have evolved rapidly. In one interesting study by Munneke et al, the efficacy of physical therapy provided by expert physiotherapists (ParkinsonNet networks) was compared to usual care. ${ }^{53}$ Whereas implementation of ParkinsonNet networks did not change health outcomes, health care costs were reduced in ParkinsonNet clusters.

The pilot study of Brefel-Courbon et al $^{54}$ assessed effectiveness of spa therapy in the management of PD. In this prospective, cross-over study, 31 PD patients were randomized to spa therapy for three weeks and a 20-week nonspa therapy. The authors concluded that direct medical costs, including radiological and laboratory tests; physician fees; drug therapy; and ancillary care, were slightly but significantly reduced in the spa period compared with the non-spa period, suggesting that spa therapy could be more effective and less expensive that conventional treatment alone. On the other hand, when the direct cost including direct and overhead costs of treatment, and consequences including: i) patient outcomes (mobility, speech and language, disability, psychological well-being, health-related quality of life); ii) career outcomes (psychological well-being, health-related quality of life, strain); and iii) social service utilization satisfaction, were measured, after using a multidisciplinary rehabilitation therapy (physical, occupational, speech and language, specialist nurse), ${ }^{55}$ facility overheads and hospital-provided transport were the main costs identified. From a patient perspective, the main consequences of the intervention were improvement of immediate outcomes for patients over four months; discovery of unmet social services needs; and high satisfaction, but no benefits for caregivers were observed.

\section{Conclusion}

In the absence of a cure, the primary goals in managing PD are to preserve functionality and HRQoL. There is no doubt that an earlier and more accurate identification of PD would allow us to identify individuals at high risk for development of PD who could participate in trials of medications designed to prevent or slow disease progression, and at the end to decrease PD related expenditures. In this regard, studies directed toward the characterization of early signs of PD (eg, the Honolulu longitudinal study) ${ }^{56}$ are needed.

In PD, treatment effects and costs accumulate over time; hence, the choice of time horizon in cost-effectiveness analysis can be particularly important. Pharmaceutical expenditures have grown rapidly in recent decades and now total nearly $10 \%$ of health care costs. Although generic drug utilization has risen substantially at the same time, the impact and cost-effectiveness of generic drugs in early PD compared to brand-names, remain unknown. Decision-analytic models evaluating interventions in PD have been carried out in different studies. However, currently available models have substantial limitations. Ideally, a comprehensive decision model for PD that can be applied to different treatment strategies should consider a large spectrum of clinically relevant outcomes and complications of the disease during a sufficiently long time horizon, including reduction of symptomatic progression; initial symptomatic improvement; or reduction of adverse effects, and improvement of HRQoL. The implementation of cost-effectiveness studies including the societal perspective should be considered as an outcome of new therapy strategies, which will be helpful to health care decision makers.

\section{Disclosure}

The authors report no conflicts of interest in this work.

\section{References}

1. Kuopio AM, Marttila RJ, Helenius H, Toivonen M, Rinne UK. The quality of life in Parkinson's disease. Mov Disord. 2000;15(2):216-223.

2. Carter JH, Stewart PG, Archbold PG, et al. Living with a person who has Parkinson's disease: the spouse's perspective by stage of disease. Parkinson's study group. Mov Disord. 1998;13(1):20-28.

3. Keränen T, Kaakkola S, Sotaniemi K, et al. Economic burden and quality of life impairment increase with severity of PD. Parkinsonism Relat Disord. 2003;9(3):163-168.

4. Janca A. Parkinson's disease from WHO perspective and a public health point of view. Parkinsonism Relat Disord. 2002;9(1):3-6.

5. Guttman M, Slaughter PM, Theriault ME, DeBoer DP, Naylor CD. Burden of parkinsonism: a population-based study. Mov Disord. 2003; 18(3):313-316.

6. Welsh M. Parkinson's disease and quality of life: issues and challenges beyond motor symptoms. Neurol Clin. 2004;22 Suppl 3:S141-S148. 
7. Dodel RC, Singer M, Kohne-Volland R, et al. The economic impact of Parkinson's disease. Pharmacoeconomics. 1998;14(3):299-312.

8. Eisenberg JM. Clinical economics. A guide to economic analysis of clinical practices. JAMA. 1989;262(20):2879-2886.

9. Markham CH, Diamond SG. Long-term follow-up of early dopa treatment in Parkinson's disease. Ann Neurol. 1986;19(4):365-372.

10. Marsden CD. Problems with long-term levodopa therapy for Parkinson's disease. Clin Neuropharmacol. 1994;17 Suppl 2:S32-S44.

11. Clarke CE. Neuroprotection and pharmacotherapy for motor symptoms in Parkinson's disease. Lancet Neurol. 2004;3(8):466-474.

12. Hoehn MM, Yahr MD. Parkinsonism: onset, progression and mortality. Neurology. 1967;17(5):427-442.

13. Eggert KM, Reese JP, Oertel WH, Dodel R. Cost effectiveness of pharmacotherapies in early Parkinson's disease. CNS Drugs. 2008;22(10): 841-860.

14. Fahn RE, Committee UD. Unified Parkinson's Disease Rating Scale. In: Fahn S, Calne D, Goldstein M, editors. Recent Developments in Parkinson's disease. Florham Park: Macmillan Healthcare information; 1987:153-163.

15. Goetz CG, Tilley BC, Shaftman SR, et al. Movement Disorder Societysponsored revision of the Unified Parkinson's Disease Rating Scale (MDS-UPDRS): scale presentation and clinimetric testing results. Mov Disord. 2008;23(15):2129-2170.

16. Karlsen KH, Tandberg E, Arsland D, Larsen JP. Health related quality of life in Parkinson's disease: a prospective longitudinal study. J Neurol Neurosurg Psychiatry. 2000;69(5):584-589.

17. Martinez-Martin P, Arroyo S, Rojo-Abuin JM, et al. Burden, perceived health status, and mood among caregivers of Parkinson's disease patients. Mov Disord. 2008;23(12):1673-1680.

18. Hagell P, Whalley D, McKenna SP, Lindvall O. Health status measurement in Parkinson's disease: validity of the PDQ-39 and Nottingham Health Profile. Mov Disord. 2003;18(7):773- 783.

19. Martínez-Martín P, Grandas F, Linazasoro G, Bravo JL. Conversion to controlled-release levodopa/carbidopa treatment and quality of life as measured by the Nottingham Health Profile. The STAR Study Group. Neurologia. 1999;14(7):338-343.

20. Brazier JE, Harper R, Jones NM, et al. Validating the SF-36 health survey questionnaire: new outcome measure for primary care. $B M J$. 1992;305(6846):160-164.

21. Longstreth WT Jr, Nelson L, Linde M, Muñoz D. Utility of the sickness impact profile in Parkinson's disease. J Geriatr Psychiatry Neurol. 1992;5(3):142-148.

22. The EuroQoL Group. EuroQol - a new facility for the measurement of health-related quality of life. The EuroQol Group. Health Policy. 1990; 16(3):199-208.

23. Siderowf A, Ravina B, Glick HA. Preference-based quality-of-life in patients with Parkinson's disease. Neurology. 2002;59(1):103-108.

24. Peto V, Jenkinson C, Fitzpatrick R. PDQ-39: a review of the development, validation and application of a Parkinson's disease quality of life questionnaire and its associated measures. J Neurol. 1998;245 Suppl 1: S10-S14.

25. Peto V, Jenkinson C, Fitzpatrick R, Greenhall R. The development and validation of a short measure of functioning and well being for individuals with Parkinson's disease. Qual Life Res. 1995;4(3): 241-248.

26. de Boer AG, Wijker W, Speelman JD, de Haes JC. Quality of life in patients with Parkinson's disease: development of a questionnaire. J Neurol Neurosurg Psychiatry. 1996;61(1):70-74.

27. Welsh M, McDermott MP, Holloway RG, et al. Development and testing of the Parkinson's disease quality of life scale. Mov Disord. 2003;18(6):637-645.

28. Schulzer M, Mak E, Calne SM. The psychometric properties of the Parkinson's Impact Scale (PIMS) as a measure of quality of life in Parkinson's disease. Parkinsonism Relat Disord. 2003;9(5):291-294.

29. McCrone P, Allcock LM, Burn DJ. Predicting the cost of Parkinson's disease. Mov Disord. 2007;22(6):804-812.
30. Dodel RC, Eggert KM, Singer MS, Eichhorn TE, Pogarell O, Oertel WH. Costs of drug treatment in Parkinson's disease. Mov Disord. 1998; 13(2):249-254.

31. Cubo E, Martínez Martin P, González M, Frades B; miembros del grupo ELEP. Impact of motor and non-motor symptoms on the direct costs of Parkinson's disease. Neurologia. 2009;24(1):15-23.

32. Leibson CL, Long KH, Maraganore DM, et al. Direct medical costs associated with Parkinson's disease: a population-based study. Mov Disord. 2006;21(11):1864-1871.

33. Siderowf AD, Holloway RG, Stern MB. Cost-effectiveness analysis in Parkinson's disease: determining the value of interventions. Mov Disord. 2000;15(3):439-445.

34. Coyle D, Barbeau M, Guttman M, Baladi JF. The economic evaluation of pharmacotherapies for Parkinson's disease. Parkinsonism Relat Disord. 2003;9(5):301-307.

35. Meara J, Bhowmick BK, Hobson P. Accuracy of diagnosis in patients with presumed Parkinson's disease. Age Ageing. 1999;28(2):99-102.

36. Scherfler C, Schwarz J, Antonini A, et al. Role of DAT-SPECT in the diagnostic work up of parkinsonism. Mov Disord. 2007;22(9):1229-1238.

37. Antonini A, Berto P, Lopatriello S, Tamma F, Annemans L, Chambers M. Cost-effectiveness of 123I-FP-CIT SPECT in the differential diagnosis of essential tremor and Parkinson's disease in Italy. Mov Disord. 2008;23(15):2202-2209.

38. Stoessl AJ. Radionuclide scanning to diagnose Parkinson disease: is it cost-effective? Nat Clin Pract Neurol. 2009;5(1):10-11.

39. Asimakopoulos P, Caslake R, Harris CE, Gordon JC, Taylor KS, Counsell C. Changes in quality of life in people with Parkinson's disease left untreated at diagnosis. J Neurol Neurosurg Psychiatry. 2008;79(6): 716-718.

40. Vossius C, Nilsen OB, Larsen JP. Health state values during the first year of drug treatment in early-stage Parkinson's disease: a prospective, population-based, cohort study. Drugs Aging. 2009;26(11):973-980.

41. Lees A. Alternatives to levodopa in the initial treatment of early Parkinson's disease. Drugs Aging. 2005;22(9):731-740.

42. Bryson HM, Milne RJ, Chrisp P. Selegiline: an appraisal of the basis of its pharmacoeconomic and quality-of-life benefits in Parkinson's disease. Pharmacoeconomics. 1992;2(2):118-136.

43. Haycox A, Armand C, Murteira S, Cochran J, François C. Cost effectiveness of rasagiline and pramipexole as treatment strategies in early Parkinson's disease in the UK setting: an economic Markov model evaluation. Drugs Aging. 2009;26(9):791-801.

44. Biglan KM, Schwid S, Eberly S, et al. Rasagiline improves quality of life in patients with early Parkinson's disease. Mov Disord. 2006;21(5): 616-623.

45. Biglan KM, Holloway RG. A review of pramipexole and its clinical utility in Parkinson's disease. Expert Opin Pharmacother. 2002;3(2): $197-210$.

46. Hoerger TJ, Bala MV, Rowland C, Greer M, Chrischilles EA, Holloway RG. Cost effectiveness of pramipexole in Parkinson's disease in the US. Pharmacoeconomics. 1998;14(5):541-557.

47. Noyes K, Dick AW, Holloway RG; for Parkinson Study Group. Pramipexole v. levodopa as initial treatment for Parkinson's disease: a randomized clinical-economic trial. Med Decis Making. 2004;24(5): $472-485$.

48. Noyes K, Dick AW, Holloway RG; for Parkinson Study Group. Pramipexole and levodopa in early Parkinson's disease: dynamic changes in cost effectiveness. Pharmacoeconomics. 2005;23(12):1257-1270.

49. Noyes K, Dick AW, Holloway RG. The implications of using USspecific EQ-5D preference weights for cost-effectiveness evaluation. Med Decis Making. 2007;27(3):327-334.

50. Iskedjian M, Einarson TR. Cost analysis of ropinirole versus levodopa in the treatment of Parkinson's disease. Pharmacoeconomics. 2003;21(2):115-127.

51. van der Marck MA, Kalf JG, Sturkenboom IH, Nijkrake MJ, Munneke M, Bloem BR. Multidisciplinary care for patients with Parkinson's disease. Parkinsonism Relat Disord. 2009;15 Suppl 3:S219-S223. 
52. Keuss SHJ, Hendriks HJM, Bloem BR, et al. KNGF Guidelines for physical therapy in Parkinson's disease. Ned Tijdschr Fysiother. 2004;114(3) Suppl. Available at: www.knfg.nl or www.cehp.nl (cited 2008 Mar 10).

53. Munneke M, Nijkrake MJ, Keus SH, et al. Efficacy of community-based physiotherapy networks for patients with Parkinson's disease: a cluster-randomised trial. Lancet Neurol. 2010;9:46-54.

54. Brefel-Courbon C, Desboeuf K, Thalamas C, et al. Clinical and economic analysis of spa therapy in Parkinson's disease. Mov Disord. 2003;18(5):578-584.
55. Gage H, Kaye J, Owen C, Trend P, Wade D. Evaluating rehabilitation using cost-consequences analysis: an example in Parkinson's disease. Clin Rehabil. 2006;20(3):232-238.

56. Abbott RD, Ross GW, White LR, et al. Excessive daytime sleepiness and subsequent development of Parkinson's disease. Neurology. 2005; 65:1442-1446.

\section{Publish your work in this journal}

ClinicoEconomics \& Outcomes Research is an international, peerreviewed open-access journal focusing on Health Technology Assessment, Pharmacoeconomics and Outcomes Research in the areas of diagnosis, medical devices, and clinical, surgical and pharmacological intervention. The economic impact of health policy and health systems organization also constitute important areas of coverage. The manuscript management system is completely online and includes a very quick and fair peer-review system, which is all easy to use. Visit http://www.dovepress.com/testimonials.php to read real quotes from published authors.

Submit your manuscript here: http://www.dovepress.com/clinicoeconomics-and-outcomes-research-journal 